\title{
Segmentation of Tropical Cyclone Eye Using Satellite Infrared Images
}

\author{
N Vanitha, C R Rene Robin
}

\begin{abstract}
The tropical cyclones are destructive weather systems and are known for their devastating effects during landfall. Cyclone tracking is one of the important tasks for the meteorologist. The eye of the tropical cyclone is the most remarkable feature. The eye of the cyclone is the roughly circular area extending over $30-65 \mathrm{~km}$ in diameter. The deepest convection is found around the eyewall for some tens of kilometers. The eye grows deeper when the cyclone becomes heavy and the winds speed grows high. In this study, the data from the 1995 - 2016 of the CIRA imagery for the tropical cyclone of the Bay of Bengal basin is analyzed and the model is developed to determine the eye of the cyclone. The segmented eye features are fed into the Rule Based Classifier which classifies the tropical cyclone images based on the presence and absence of the eye.
\end{abstract}

Index Terms: segmentation, image processing, classification

\section{INTRODUCTION}

The cyclone is the deadliest disaster which causes huge damage to life and property. They have different names such as a hurricane, typhoon, tropical cyclone, storm, tornado based on the region. The strong tropical cyclone has a calm region at the center is known as the eye. The eye is a circular area with $30-65 \mathrm{~km}$ in diameter. As the cyclone becomes severe the eye of the cyclone grows deeper. The eye portion is formed when the cyclone becomes worst and its wind speed becomes high. The wind shear is high at the eye portion. The formation and location of the eye have a large impact on predicting the track of the cyclone. The tropical cyclone may or may not contain an eye feature during the development stage but its location provides key information for the cyclone track forecasting. This study investigates 30 cyclones that occurred during the period 1995 - 2016 that formed over the Bay of Bengal sub-basin of the North Indian Ocean. In this paper, the image is enhanced by applying filtering techniques and then the major features such as eye and edges are enhanced. The Sobel filter is used to enhance the resolution of the image for identifying the tropical cyclone eye feature. These features are envisaged to train the Rule-Based Classifier which identifies the presence or absence of eye on a tropical cyclone.

Revised Manuscript Received on July 22, 2019

N Vanitha, Research Scholar, Anna University, Chennai 600025, India Assistant Professor, Department of Computer Applications, New Prince Shri Bhavani College of Engineering and Technology, Chennai 600073,India

C R Rene Robin, Professor, Department of Computer Science and Engineering, Jerusalem College of Engineering, Chennai 600100, India

\section{RELATED WORK}

The Bay of Bengal accounts for $7 \%$ of tropical cyclones all over the world [1]. The estimation of the eye of the tropical cyclone is vital for the forecasters. Wong et al., [2] developed the motion field analysis method to locate the TC eyes. The Doppler velocity is computed and ROI is generated by applying the motion estimation techniques. Matching a high reflectivity area to the outer circle and low reflectivity area to the inner circle of the spiral segment is done using the fitness function [3]. The powerful wind generated by the tropical cyclone causes serious hazards during landfall. The impact of the vertical wind to the TC center is computed using wavelet analysis by analyzing five typhoon cases [4]. The central location of the typhoon eye is estimated using SAR and MTSAT images [5]. An exhaustive list of parameters is used for the classification using Identification Tree (IDT) [6].

\section{METHOD}

In this study we propose thresholding based tropical cyclone eye segmentation and Rule Based Classification using satellite IR images. Figure 1 shows the architecture of the tropical cyclone eye detection and classification system.

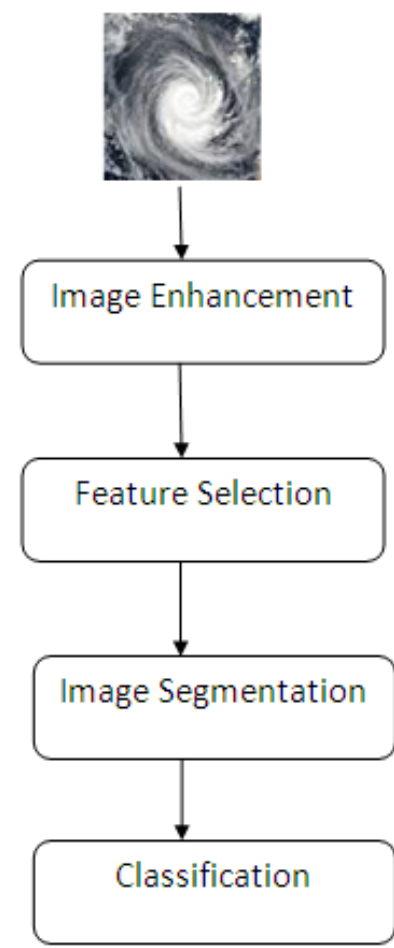

Fig.1 Stages of TC eye detection and classification system.

A. Image enhancement 


\section{Segmentation of Tropical Cyclone Eye Using Satellite Infrared Images}

The main objective of the enhancement technique is to process the image so that the result is more suitable for the specific application than the original image. The image enhancement techniques are used to filter out noise, to emphasize the low, high or directional spatial frequency components, etc. In an image, a pixel's neighborhood is defined by some set of relatively located pixels. Some algorithms are applied to the values of the neighbor, then the result values are mapped to output image - this process of neighborhood operation is known as Filtering. Filtering is one of Spatial domain methods are procedures that operate directly on these pixels. The tropical cyclone image is convolved using the Sobel filter which has two kernels in the $\mathrm{x}$ and $\mathrm{y}$ directions respectively. The Sobel kernels respond maximally to the edges which when applied to the input image produces the gradient components Gx and Gy. the gradient magnitude is given by

$$
|\mathrm{G}|=\sqrt{G \times 2+G y 2}
$$

The angle of orientation of the edges forms the spatial gradient which is given by

$$
\Phi=\arctan \left(\frac{G x}{G y}\right)
$$

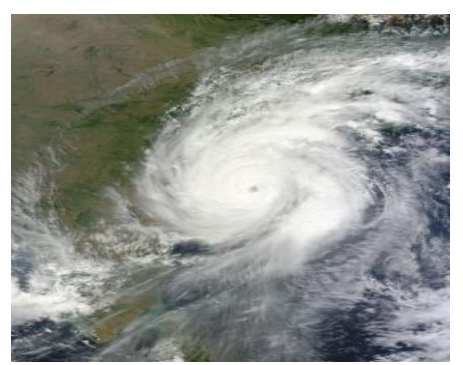

a) Orissa Super TC

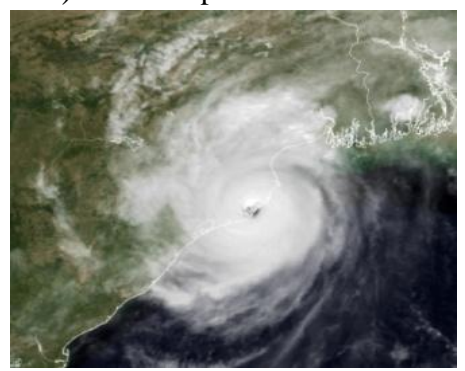

b) Hud Hud TC

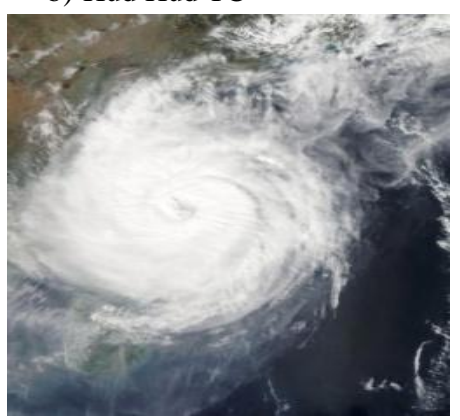

c) Thane TC

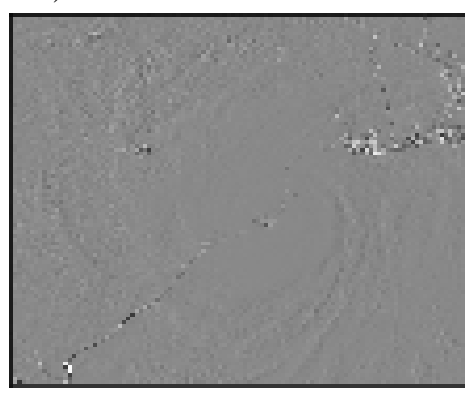

d) Sobel X of Orissa Super TC

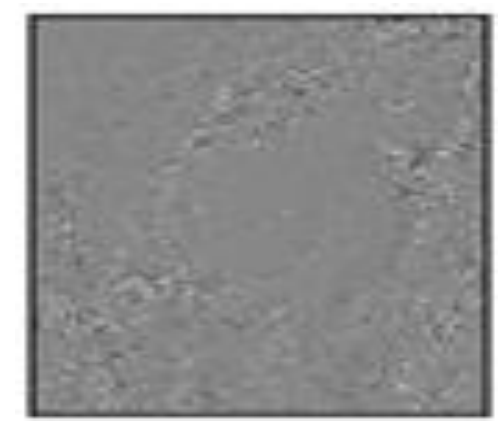

e) Sobel X of Hud Hud TC

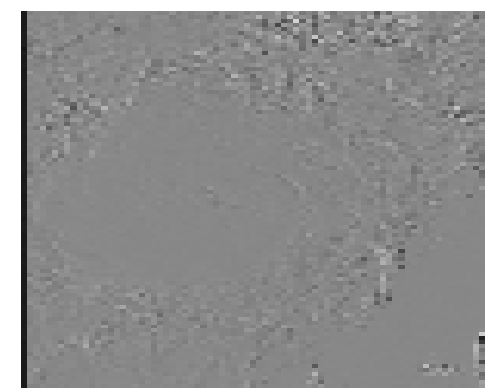

f) Sobel X of Thane TC

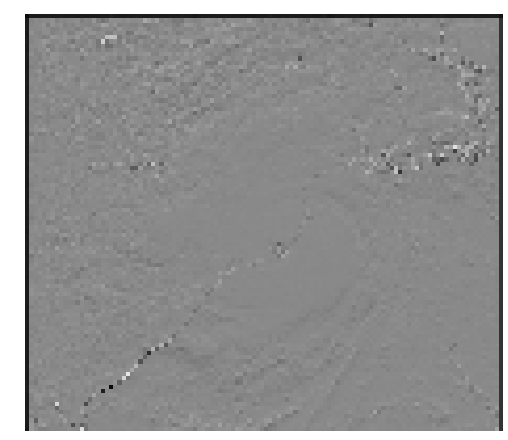

g) Sobel Y of OrissaSuper TC

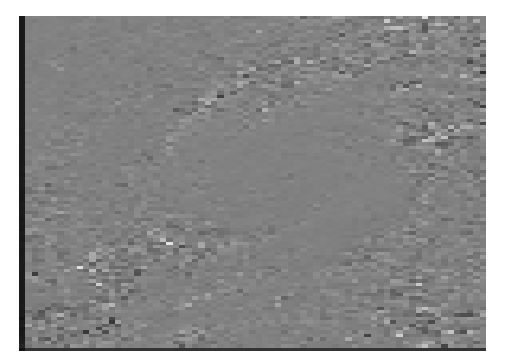

h) Sobel Y of Hud Hud TC

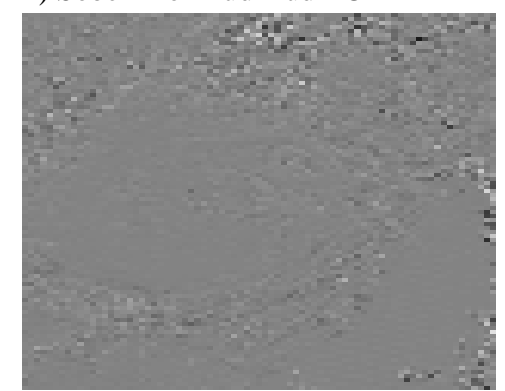
TC

i)Sobel Y of Thane

Fig. 2 Edge detection by applying Sobel filter

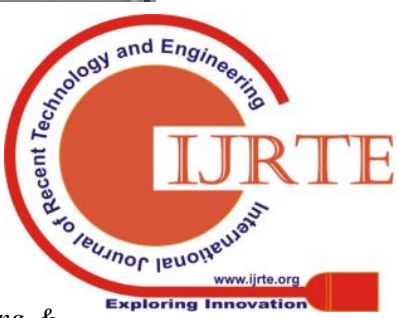




\section{B. Feature selection}

The textural feature represent the spatial distribution of the images plays a vital role in many areas such as medical imaging, remote sensing, video processing. The image features help to find the region of common property in an image and these regions form the segment of the image. These features form the basis for the segmentation process. The different features used in this proposed system are tropical cyclone center eye_area_ratio, eye_density, and tc_curvature. The first feature eye_area_ratio is computed by dividing the total number of pixels in the eye area with the total number of pixels of the whole image.

$$
\text { eye_area_ratio }=\frac{\text { Area of } \text { eye }}{\text { Area of cyclone }} * 100
$$

The second feature is based on the darkness of the tropical cyclone eye region. A vector of RGB component is computed which gives the average intensity values of the pixels of particular regions.

$$
\text { eye_density }=\frac{\sum_{i=1}^{n} \text { intensity(i) }}{\text { Area of } \operatorname{\theta ys}}
$$

The tc_curvature is given by the rate of change of corner points of the image.

$$
\Phi(\mathrm{t})=\tan ^{-1}\left(\frac{y(t)}{x(t)}\right)
$$
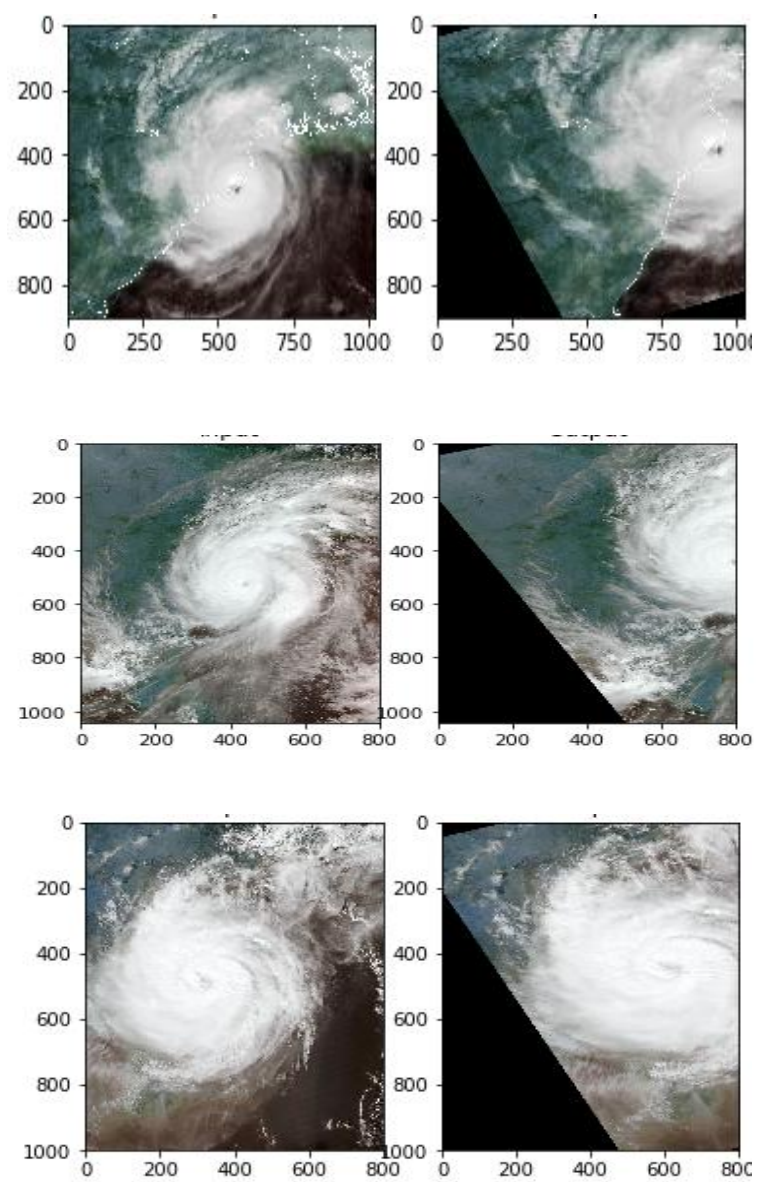

Fig. 3 Image rotation is done to accomplish image processing task such as feature extraction. Original and rotated images of Orissa Super cyclone, Hud Hud and Thane

\section{Image segmentation}

The tropical cyclone images are partitioned into regions based on the intensity values. The regions are grown by using the intensity values and by applying the Red, green and Blue channels. By defining the threshold these intensity values are used to differentiate the object point and the background point. The region growing process is constrained by the threshold values to get the segmented image. The thresholds have been computed experimentally for the features eye_area_ratio, density, and tc_curvature are as $\mathrm{T} 1=0.2, \mathrm{~T} 2=116 \mathrm{~T} 3=60^{\circ}$ respectively.
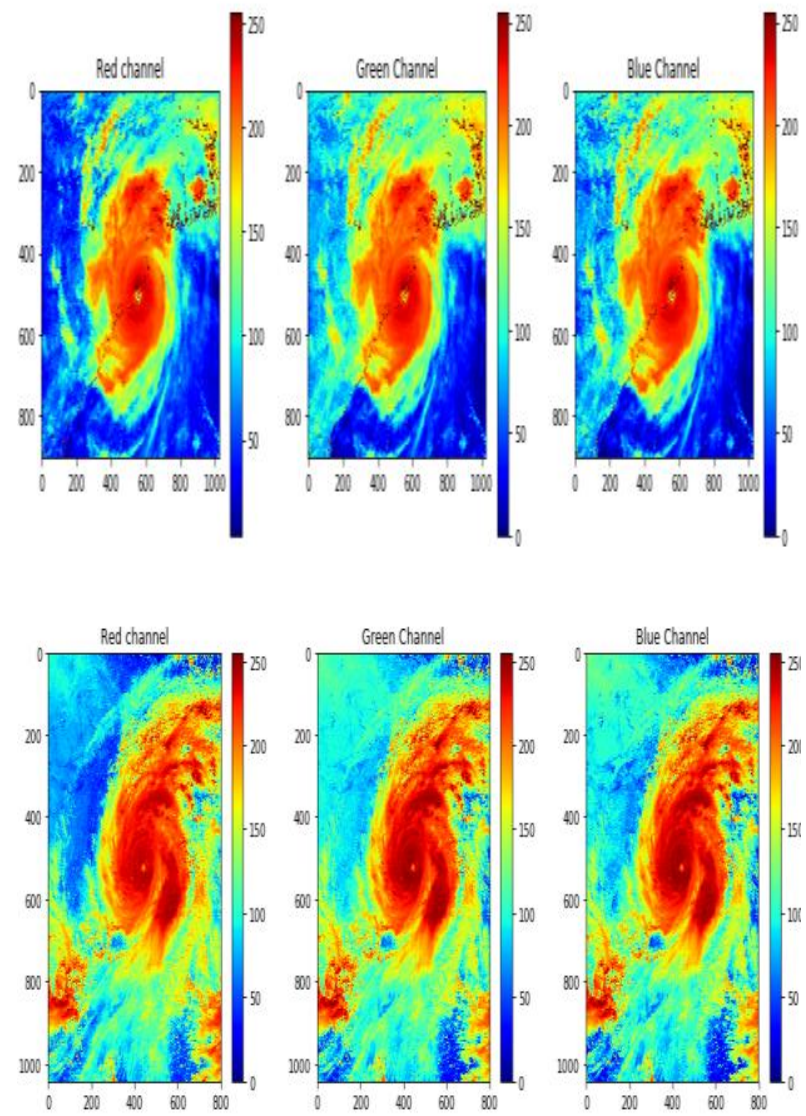

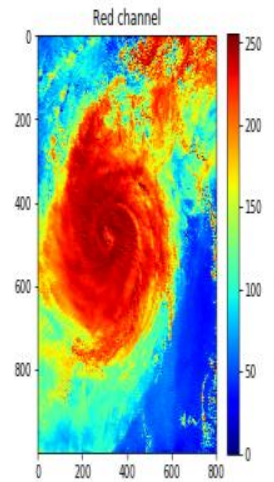

a)

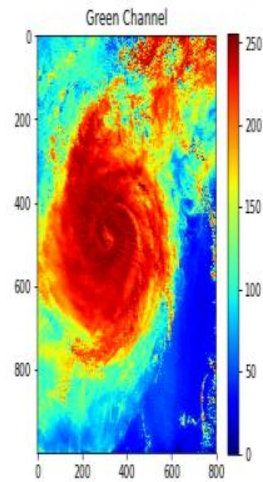

b)

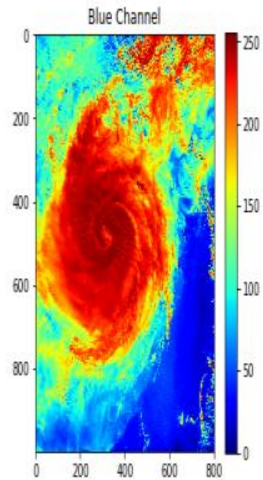

c)

Fig. 4 (a) Red channel segmentation (b) Green channel segmentation (c) Blue channel segmentation

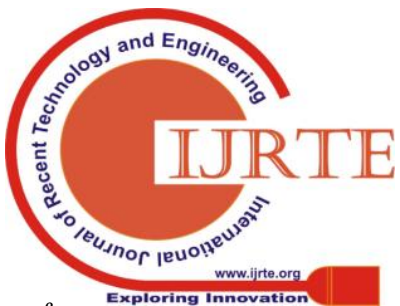



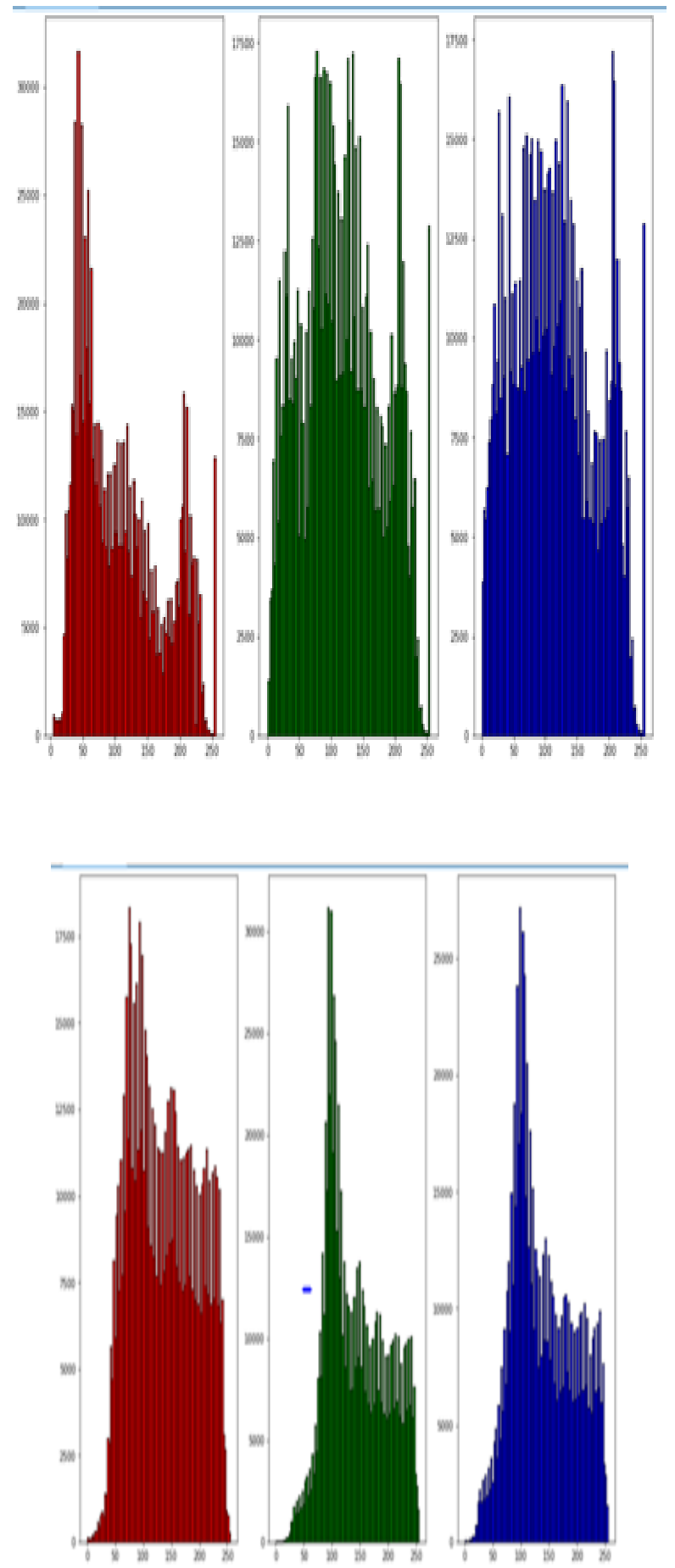

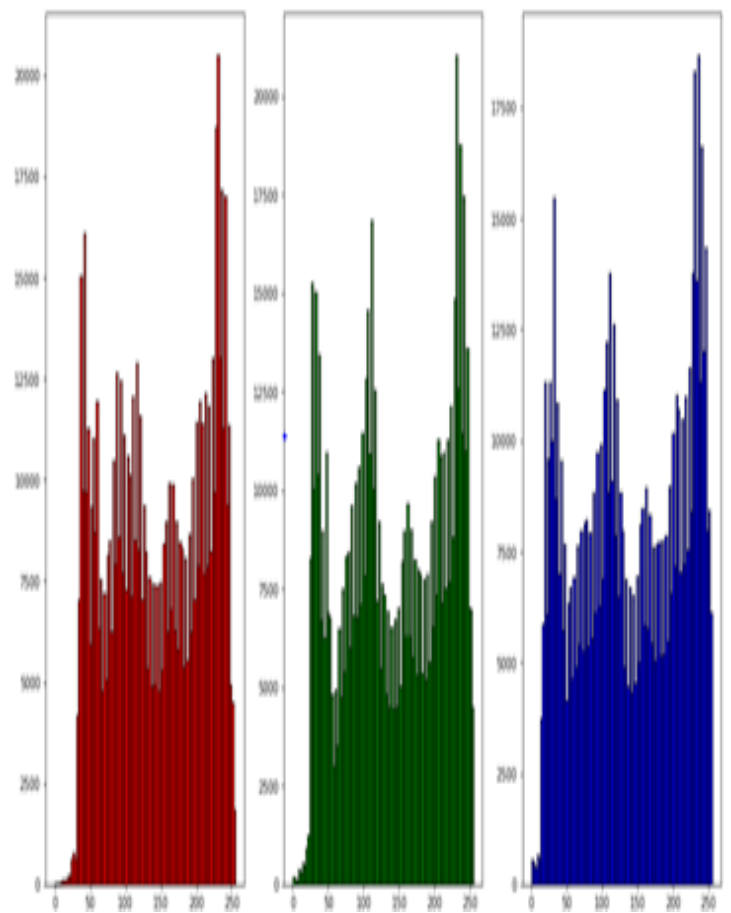

Fig. 5 Histogram representing pixel distribution on RGB channel of three cyclonic storms.

\section{Rule Based Classification}

Rule Based method is a widely used classification technique that uses a collection of IF-THEN rules. The feature threshold of the training images is used to train the Rule Based classifier. After feature extraction and segmentation a rule based classification system is constructed which classifies the images based on the presence and absence of the eye feature. The learned rules are as follows

Rule 1: r1 = eye_area_ratio.

If $\mathrm{r} 1 \geq \mathrm{T} 1$ then the $\mathrm{TC}$ has an eye else $\mathrm{TC}$ has no eye

Rule 2: $\mathrm{r} 2=$ eye_density

If $\mathrm{r} 2 \geq \mathrm{T} 2$ then the $\mathrm{TC}$ has an eye else $\mathrm{TC}$ has no eye

Rule 3: $r$ 3 = tc_curvature

If $\mathrm{r} 3 \geq \mathrm{T} 3$ then the $\mathrm{TC}$ has an eye else $\mathrm{TC}$ has no eye

The classification based on the presence and absence of the eye feature is achieved with the following algorithm

If ( $\mathrm{r} 1 \geq \mathrm{T} 1)$ then the TC has an eye

Elseif( $(2 \geq \mathrm{T} 2)$ then the TC has an eye

Elseif(r $3 \geq \mathrm{T} 3)$ then the TC has an eye

Else the TChas no eye.

\section{CONCLUSION}

In this paper, we presented a new technique for the detection of an eye on the tropical cyclone. A different set of features such as eye_area_ratio, eye_density, tc_curvature are identified. Based on the features and by applying the RGB channels the regions are made grown.

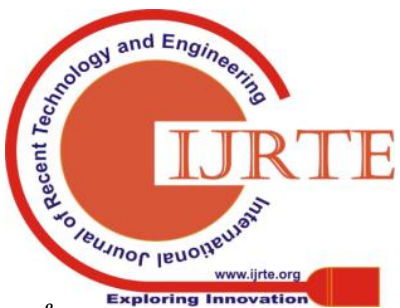


Based on the threshold value the segmentation of tropical cyclone eye is done. The rule based classifier classifies tropical cyclone images based on the presence and absence of an eye.

\section{REFERENCES}

1. U C Mohanty, "Tropical cyclone in the Bay of Bengal and deterministic Method for Prediction of their trajectories", Springer, Sadhana, vol. 19, pp. 567 - 582, 1994.

2. Ka Yan Wong, Chi Lap Yip and Ping Wah Li, "A novel algorithm for automatic tropical cyclone eye fix using Doppler radar data", RMets, Meteorological Applications, vol. 14, pp. 49-59, 2007.

3. Ka Yan Wong, Chi Lap Yip and Ping Wah Li, "Automatic tropical cyclone eye fix using genetic algorithm", Elsevier, Expert Systems with Applications, vol. 34, pp.643 - 656, 2008.

4. Xuezhu Lv ; Xiaofeng Li ; Xiaofeng Yang ; William Pichel ; Xuan Zhou ; Yuguang Liu, "The impact of vertical wind shear on the hurricane eye tilt at the sea and cloud levels" , 2013 - IEEE International Geoscience and Remote Sensing Symposium IGARSS, IEEE, pp. 566 - 569.

5.Y. Cheng, S. Huang, A.K. Liu, C. Ho, and N. Kuo, "Observation of typhoon eyes on the sea surface using multi-sensors", Remote Sensing of Environment, 123, 434-442, 2012.

6. Jyotshna, Dongardive, Agnes Xavier, Kavita Jain and Siby Abraham, "Classification and Rule-Based Approach to Diagnose Pulmonary Tuberculosis", International Conference on Advances in Computing and Communications, Springer, pp. 328 -339. 